PAPER

\title{
A Supervised Classification Approach for Measuring Relational Similarity between Word Pairs
}

\author{
Danushka BOLLEGALA $^{\dagger a)}$, Yutaka MATSUO ${ }^{\dagger}$, Nonmembers, and Mitsuru ISHIZUKA ${ }^{\dagger}$, Member $^{\circ}$
}

\begin{abstract}
SUMMARY Measuring the relational similarity between word pairs is important in numerous natural language processing tasks such as solving word analogy questions, classifying noun-modifier relations and disambiguating word senses. We propose a supervised classification method to measure the similarity between semantic relations that exist between words in two word pairs. First, each pair of words is represented by a vector of automatically extracted lexical patterns. Then a binary Support Vector Machine is trained to recognize word pairs with similar semantic relations to a given word pair. To train and evaluate the proposed method, we use a benchmark dataset that contains 374 SAT multiple-choice word-analogy questions. To represent the relations that exist between two word pairs, we experiment with 11 different feature functions, including both symmetric and asymmetric feature functions. Our experimental results show that the proposed method outperforms several previously proposed relational similarity measures on this benchmark dataset, achieving an SAT score of 46.9. key words: relational similarity, supervised classification, support vector machines, word analogies
\end{abstract}

\section{Introduction}

Relational similarity can be defined as the correspondence between semantic relations that exist between words. For example, the semantic relation, $\mathbf{X}$ is a large $\mathbf{Y}$ holds between the words in the word pair (lion, cat) and (ostrich, bird), because lion is a large cat, whereas ostrich is a large bird. Here, we use variables $\mathbf{X}$ and $\mathbf{Y}$ as placeholders for words between which a relation exists. Consequently, the two word pairs, (lion, cat) and (ostrich, bird), are considered to be relationally similar. If four words $A, B, C$, and $D$ form a proportional analogy $A: B:: C: D$, then we can observe a high degree of relational similarity between the two word pairs $(A, B)$ and $(C, D)$.

Relational similarity measures are useful for numerous tasks in natural language processing such as classification of semantic relations in noun-modifier pairs, word sense disambiguation (WSD) and automatic thesaurus generation. Noun-modifier pairs such as flu virus, storm cloud, expensive book, etc. are frequent in English language. In fact, WordNet contains more than 26,000 noun-modifier pairs. Natase and Szpakowicz [1] classified noun-modifiers into five classes according to the relations between the noun and the modifier. Turney [2] used a relational similarity measure to compute the similarity between noun-modifier pairs and classify them according to the semantic relations that hold between a noun and its modifier. In WSD [3] identifying the

\footnotetext{
Manuscript received May 6, 2011.

†The authors are with The University of Tokyo, Tokyo, 1138656 Japan.

a)E-mail: danushka@iba.t.u-tokyo.ac.jp

DOI: 10.1587/transinf.E94.D.2227
}

various relations that hold between an ambiguous word and its context is vital. For example, the word "plant" can refer to an industrial plant or a living organism. If the word "food" appears in the immediate context of "plant", then a typical WSD approach is to compare the attributional similarity between "food" and "industrial plant" to that of "food" and "living organism" and to select the sense with higher attributional similarity. Considering the fact that industrial plants often produce food and living orgasms often serve as food, the decision may not be very clear. However, if we can identify the relation between "food" and "plant" as "food for the plant" then it strongly suggests that the plant is a living organism. On the other hand, a relation such as "food at the plant" suggests the plant to be an industrial plant.

To accurately measure the relational similarity between two word pairs, we must overcome several challenges. First, the relations themselves are only implicitly stated by a word pair. Therefor, we must first extract the relation that exists between the two words in each of the word pairs. For example, from the word pair (ostrich, bird), we must first identify the relation $\mathbf{X}$ is a large $\mathbf{Y}$. Second, there might exist more than one relation between two words. For example, in addition to the relation $\mathbf{X}$ is a large $\mathbf{Y}$, there exists the relation $\mathbf{X}$ is a flightless $\mathbf{Y}$ between the two words ostrich and bird. Third, we must identify how much each relation contributes to the relational similarity between two word pairs. For example, a general relation such as $\mathbf{X}$ and $\mathbf{Y}$ which holds between many words might contribute less towards relational similarity, whereas a specific relation such as $\mathbf{X}$ is a large $\mathbf{Y}$ might contribute more. However, it is not known a priori as to how much each relation contributes to the relational similarity between two given word pairs. In this paper, we follow a supervised machine learning approach to measure the relational similarity between two given word pairs.

We model the problem of detecting relational similarity as a binary classification problem in which given two word pairs, $(A, B)$ and $(C, D)$ the classifier must return a positive or a negative decision depending on whether the word pair $(A, B)$ is relationally similar to the word pair $(C, D)$ or otherwise. We use Support Vector Machine (SVM)s [4] as the binary classifier and converts the distance from the decision hyperplane into a calibrated posterior probability, thereby enabling us to measure degree of relational similarity between two given word pairs. Following previous work on relational similarity, we represent the implicit semantic relations that exist between two words using lexical patterns. Specifically, we use the method proposed by Bollegala et 
al. [5] to extract lexical patterns to represent semantic relations that exist between two given words using text-snippets returned from a Web search engine. Snippets provide useful information about the relations that hold between words. For example, Google ${ }^{\dagger}$ returns the snippet ...the ostrich is the largest bird in the world and can be found in South Africa... for the conjunctive query ostrich AND bird. This snippet alone suggests that ostrich is a large bird. We automatically extract lexical patterns that describe the relations implied by the two words in a word pair and compute the relational similarity using a machine learning approach. Moreover, by using snippets, we can obviate the need to download Web pages which can be time consuming if those pages are large.

\section{Related Work}

Scholastic Aptitude Test (SAT) word analogy questions have been used for evaluating relational similarity measures. An SAT word analogy question consists of a word pair (which we designate as the question word pair) and five candidate answer word pairs (which we designate as the candidate word pairs). Only one of the candidate word pairs is analogous to the source word pair in an SAT word analogy question. An examinee is required to select the analogous word pair to the question word pair. An example is shown in Fig. 1. The relatively low average human score of $57 \%$ reported for the SAT word analogy questions indicates that detecting the correct word pair among a given set of candidates is difficult for even humans.

Turney et al. [6] combined 13 independent modules by considering the weighted sum of the outputs of each individual module to solve SAT analogy questions. The best performing individual module was based on Vector Space Model (VSM). In the VSM approach to measuring relational similarity [7], first a vector is created for a word-pair $(X, Y)$ by counting the frequencies of various lexical patterns containing $X$ and $Y$. In their experiments they used 128 manually created patterns such as " $X$ of $Y$ ", " $Y$ of $X$ ", " $X$ to $Y$ " and " $Y$ to $X$ ". These patterns are then used as queries to a search engine and the number of hits for each query is used as elements in a vector to represent the word pair. Finally, the relational similarity is computed as the cosine of the angle between the two vectors representing each word-pair. This VSM approach achieves a score of $47 \%$ on collegelevel multiple-choice SAT analogy questions. A SAT analogy question consists of a target word-pair and five choice word-pairs. The choice word-pair that has the highest relational similarity with the target word-pair in the question is selected by the system as the correct answer.

Question: Ostrich is to Bird as
a. Cub is to Bear
b. Lion is to Cat
c. Ewe is to Sheep
d. Turkey is to Chicken
e. Jeep is to Truck

Fig. 1 An SAT word analogy question. Choice (b) is the answer.
Turney [2] proposes Latent Relational Analysis (LRA) by extending the VSM approach in three ways: a) lexical patterns are automatically extracted from a corpus, b) the Singular Value Decomposition (SVD) is used to smooth the frequency data, and c) synonyms are used to explore variants of the word-pairs. LRA achieves a score of $56 \%$ on SAT analogy questions. Both VSM and LRA require a large number of search engine queries to create a vector representing a word-pair. For example, with 128 patterns, VSM approach requires at least 256 queries to compute relational similarity. LRA considers synonymous variants of the given word pairs, thus requiring even more search engine queries. In contrast, our proposed method matches numerous patterns among the text snippets retrieved from a Web search engine for the two words in a word pair. We do not search using each extracted pattern as done in VSM and LRA methods. Therefore, the number of Web search queries does not increase with the number of patterns extracted. This enables us to represent relations using a large number of patterns.

Veale [8] proposed a relational similarity measure based on taxonomic similarity in WordNet. He evaluates the quality of a candidate analogy $A: B:: C: D$ by looking for paths in WordNet, joining $A$ to $B$ and $C$ to $D$. Then, the relational similarity is computed based on the similarity between the $A: B$ paths and $C: D$ paths. If the set of WordNet relations that connects $A$ to $B$ and the set of WordNet relations that connects $C$ to $D$ has many relations in common (i.e. a high overlap between the two sets), then the relational similarity between two word pairs $(A, B)$ and $(C, D)$ is high. His method achieves a score of 43 on the SAT word analogy questions. However, the dependence on the WordNet means that this method cannot compute the relational similarity when the word pairs have words that do not appear in the WordNet.

Bollegala et al. [9] proposed a supervised metric learning approach to solve SAT word analogy questions. First, they use SAT questions to induce pairwise distance constraints between word pairs. Next, a Mahalanobis distance metric is learnt from the pairwise constraints. They use the information theoretic metric learning (ITML) algorithm to learn the Mahalanobis distance metric. Evaluations are conducted both on SAT word analogy questions as well as on a novel dataset (ENT dataset) that consists of named entities frequently found on the Web. They use lexical patterns to represent the relations that exist between two words. They show that by clustering the lexical patterns that represent the same semantic relation, one can improve the accuracy of the relational similarity measurement. However, both clustering and learning distance metrics in high dimensional lexical pattern space is computationally expensive.

\section{Method}

The proposed method can be described in two main steps: identifying the implicit relations between the two words in

${ }^{\dagger}$ http://google.com 
...lion, a large heavy-built social cat of open rocky areas in Africa ...

Fig. 2 A snippet returned by Google for the query "lion $* * * * * * *$ cat".

each word pair and learning a binary classification model to recognize relationally similar word pairs. Next, we describe each of those steps in detail.

\subsection{Pattern Extraction}

We use the subsequence lexical pattern extraction algorithm first proposed by Bollegala et al. [9] for the task of representing the implicit semantic relations that exist between two words. For completeness, we briefly describe this algorithm here. For further details refer the original paper.

To identify the implicit relations between two words $\mathbf{X}$ and $\mathbf{Y}$, we first query a web search engine using the phrasal query " $\mathbf{X} * * * * * * * \mathbf{Y}$ ". Here, the wildcard operator "**" would match any word or nothing. This query retrieves snippets that contain both $\mathbf{X}$ and $\mathbf{Y}$ within a window of 7 words. For example, Google returns the snippet shown in Fig. 2 for the word pair (lion, cat). We use PrefixSpan (i.e., prefixprojected sequential pattern mining) [10] algorithm to extract frequent subsequences from snippets that contain both $\mathbf{X}$ and $\mathbf{Y}$. PrefixSpan extracts all word subsequences which occur more than a specified frequency in snippets. We select subsequences that contain both query words (eg. lion and cat) and replace the query words respectively with variables $\mathbf{X}$ and $\mathbf{Y}$ to construct lexical patterns. For example, some of the patterns extracted by the proposed algorithm from the snippet in Fig. 2 are $\mathbf{X}$ a large $\mathbf{Y}, \mathbf{X}$ a large $\mathbf{Y}$ of and $\mathbf{X}, a$ large social $\mathbf{Y}$. PrefixSpan algorithm is particularly attractive for the current task because it can efficiently extract a large number of lexical patterns. Moreover, its ability to skip words when creating patterns enables us to capture relations between words that appear at a distance in snippets.

\subsection{Pattern Selection}

We used the pattern extraction algorithm described in Sect. 3.1 to extract lexical patterns for 374 SAT multiplechoice analogy questions. This dataset was first proposed by Turney and Littman [7] as a benchmark dataset to evaluate relational similarity measures. Generally, there are six word pairs in each question (i.e. one word pair for the question and five choices) which amounts to 2176 (cf. some questions have less than 5 candidates) word-pairs. For each word pair, using Yahoo BOSS Search API ${ }^{\dagger \dagger}$ we download 7000 snippets on average. Yahoo BOSS Search API allows only 1000 snippets to be retrieved for a single query. To overcome this limitation, we issue multiple contextual queries by varying the number of asterisks between the two words in a query. This process is repeated with the two words inter-changed. Finally, duplicate snippets are removed from the retrieved search results.
Table 1 Most frequent patterns in the corpus.

\begin{tabular}{|c|c|l|}
\hline Rank & Frequency & Lexical Pattern \\
\hline 1 & 272892 & Y and X \\
2 & 240802 & X and Y \\
3 & 91720 & X of Y \\
4 & 78125 & Y or X \\
5 & 78011 & X the Y \\
6 & 74428 & Y to X \\
7 & 71260 & X or Y \\
8 & 65470 & Y of X \\
9 & 63986 & $\mathbf{X}$ to $\mathbf{~}$ \\
10 & 55062 & $\mathbf{X ~ Y ~ s}$ \\
11 & 52106 & $\mathbf{X ~ Y . ~}$ \\
12 & 44049 & $\mathbf{X}, \mathbf{Y}$ \\
13 & 42708 & Y the X \\
14 & 40434 & Y X s \\
15 & 37608 & $\mathbf{X ~ Y ,}$ \\
16 & 36678 & $\mathbf{X}$ of the Y \\
17 & 34725 & Y, X \\
18 & 33851 & Y.X \\
19 & 33331 & Y and X. \\
20 & 31484 & Y X. \\
\hline
\end{tabular}

In addition to the snippets, we also select the titles of pages (also returned by the Yahoo BOSS Search API alongside with the search results) for extracting patterns. The corpus of snippets and titles downloaded from all SAT word pairs contains 412, 110,644 tokens. We run the pattern extraction algorithm described in the previous section on this corpus and extract 12,712,608 lexical patterns. However, this set of patterns is very sparse and most patterns occur only a few times in the corpus. Consequently, we select patterns that occur at least 50 times in the corpus for the remainder of the experiments described in this paper. The selected set contains 48, 253 lexical patterns. Top ranked 10 patterns are shown in Table 1 alongside with their frequencies in the corpus.

\subsection{Training}

For given two pairs of words $(A, B)$ and $(C, D)$, we create a feature vector using the patterns selected in Sect. 3.1. First, we record the frequency of occurrence of each selected pattern in snippets for each word pair. We call this the pattern frequency. It is a local frequency count, analogous to term frequency in information retrieval [11]. Second, we combine the two pattern frequencies of a pattern (i.e., frequency of occurrence in snippets for $(A, B)$ and that in snippets for $(C, D))$ using various feature functions to compute the feature-values for training. The different feature functions experimented in the paper are explained in Sect.4.1.

We model the problem of computing relational similarity as a one of identifying analogous and non-analogous word pairs, which can be solved by training a binary classifier. Using SAT analogy questions as training data, we train a two-class support vector machine (SVM) as follows. From each question in the dataset, we create a positive training instance by considering $(A, B)$ to be the word pair for

\footnotetext{
${ }^{\dagger \dagger} h$ ttp://developer.yahoo.com/search/boss/
} 
the question (i.e. stem) and $(C, D)$ to be the word pair for the correct answer. Likewise, a negative training instance is created from a question word pair and one of the incorrect answers.

The trained SVM model can then be used to compute the relational similarity between two given word pairs $(A, B)$ and $(C, D)$ as follows. First, we represent the two word-pairs by a feature vector $\boldsymbol{F}$ of pattern frequencybased features. Second, we define the relational similarity $\operatorname{RelSim}((A, B),(C, D))$ between the two word-pairs $(A, B)$ and $(C, D)$ as the posterior probability $\operatorname{Prob}(\boldsymbol{F} \mid$ analogous $)$ that feature vector $F$ belongs to the analogous-pairs (positive) class,

\section{$\operatorname{RelSim}((A, B),(C, D))=\operatorname{Prob}(\boldsymbol{F} \mid$ analogous $)$.}

Being a large margin classifier, the output of an SVM is the distance from the decision hyper-plane. For the purpose of solving SAT questions, we can directly use the distance from the decision hyper-plane and rank the candidate answers. However, distance from the decision hyper-plane is not a calibrated posterior probability that lies between $[0,1]$ range. We use sigmoid functions to convert this uncalibrated distance into a calibrated posterior probability using the method proposed by Platt [12].

\section{Experiments and Results}

For the experiments in this paper we used the 374 SAT college-level multiple-choice analogy questions dataset which was first proposed by Turney et al. [6]. We compute the total score for answering SAT questions as follows,

$$
\text { score }=\frac{100 \times \text { no. correct }}{\text { total no. of questions }} .
$$

\subsection{Feature Functions}

In previous sections we described a method to represent a word pair using a pattern frequency vector. However, we are interested in measuring relational similarity between two word pairs. Therefore, we must somehow represent two word pairs using a single feature vector. It is not obvious how to construct a single feature vector to represent two word pairs using pattern frequencies that appear in two separate feature vectors representing each word pair.

Bollegala et al. [5] defined several functions to combine features from two vectors to construct a feature vector for two word pairs. However, they only evaluated symmetric functions because previous results in psychological experiments investigating similarity between words suggest that although similarity is an asymmetric phenomenon, the degree of asymmetry is less than 5\%. However, this does not limit us to use only symmetric feature functions. We extend the set of feature functions proposed in [5] to include asymmetric feature functions and evaluate their effect empirically. Next, we describe each of the feature functions we investigate in this paper.
Let us assume the frequency of a pattern $v$ in two word pairs $(A, B)$ and $(C, D)$ to be $f_{A B}$ and $f_{C D}$, respectively. We compute the value assigned to the feature corresponding to pattern $v$ in the feature vector that represents the two word pairs $(A, B)$ and $(C, D)$ using the following four symmetric feature functions.

1. $\left|f_{A B}-f_{C D}\right|$ : The absolute value of the difference of pattern frequencies is considered as the feature value.

2. $\left(f_{A B}-f_{C D}\right)^{2}$ : The square of the difference of pattern frequencies is considered as the feature value.

3. $f_{A B}+f_{C D}$ : The sum of pattern frequencies is considered as the feature value.

4. $f_{A B} \times f_{C D}$ : The product of the pattern frequencies is considered as the feature value.

5. JS divergence: Ideally, if two word pairs are analogous we would expect to see similar distributions of patterns in each word pair. Consequently, the closeness between the pattern distributions can be regarded as an indicator of relational similarity. We define a feature function based on Jensen-Shannon divergence [13] as a measure of the closeness between pattern distributions. Jensen-Shannon (JS) divergence $D_{J S}(P \| Q)$, between two probability distributions $P$ and $Q$ is given by,

$$
D_{J S}(P \| Q)=\frac{1}{2}\left(D_{K L}(P \| M)+D_{K L}(Q \| M)\right) .
$$

Here, $M=(P+Q) / 2$ and $D_{K L}$ is Kullback-Leibler divergence, which is given by,

$$
D_{K L}(P \| Q)=\sum_{v} P(v) \log \frac{P(v)}{Q(v)} .
$$

Here, $P(v)$ denotes the normalized pattern frequency of a pattern $v$ in the distribution $P$. Pattern frequencies are normalized s.t. $\sum_{v} P(v)=1$ by dividing the frequency of each pattern by the sum of frequencies of all patterns. We define the contribution of each pattern towards the total JS-divergence in Formula 2 as its feature value, $J S(v)$. Substituting Formula 3 in 2 and collecting the terms under summation, we derive $J S(v)$ as,

$$
J S(v)=\frac{1}{2}\left(p \log \frac{2 q}{p+q}+q \log \frac{2 p}{p+q}\right) .
$$

Here, $p$ and $q$ respectively denote the normalized pattern frequencies of $f_{A B}$ and $f_{C D}$.

All of the above-described feature functions are symmetric in their arguments (i.e. we obtain the same feature value even if we reverse the two feature vectors given as arguments for the feature function). Next, we define asymmetric feature functions.

1. $f_{A B} / f_{C D}$ : The ratio between the frequency of pattern $v$ in the two vectors is taken as the feature value. If $f_{C D}$ is zero, then we set the feature value to zero.

2. $f_{C D} / f_{A B}$ : Same as above, except we consider the re- 
Table 2 Effect of feature functions and kernel functions.

\begin{tabular}{|l|c|c|c|c|c|}
\hline Function & Linear & deg=2 & deg=3 & RBF & Sigmoid \\
\hline \hline$\left|f_{A B}-f_{C D}\right|$ & 24 & 28 & 28 & 20 & 18 \\
$\left(f_{A B}-f_{C D}\right)^{2}$ & 22 & 20 & 24 & 24 & 18 \\
$f_{A B}+f_{C D}$ & 26 & 32 & 36 & 10 & 12 \\
$f_{A B} \times f_{C D}$ & 44 & 50 & 46 & 58 & 50 \\
JS divergence & 24 & 26 & 28 & 24 & 22 \\
\hline \hline$f_{A B} / f_{C D}$ & 22 & 12 & 14 & 16 & 14 \\
$f_{C D} / f_{A B}$ & 26 & 22 & 14 & 16 & 14 \\
$f_{A B}-f_{C D}$ & 26 & 26 & 24 & 20 & 22 \\
$f_{C D}-f_{A B}$ & 26 & 26 & 24 & 20 & 22 \\
$D_{K L}\left(f_{A B} \| f_{C D}\right)$ & 46 & 50 & 52 & 36 & 20 \\
$D_{K L}\left(f_{C D} \| f_{A B}\right)$ & 22 & 20 & 16 & 16 & 8 \\
\hline
\end{tabular}

versed ratio. If $f_{A B}$ is zero, then we set the feature value to zero.

3. $f_{A B}-f_{C D}$ : We subtract frequencies of patterns in each word pair and take it as the feature value.

4. $f_{C D}-f_{A B}$ : Same as above, except we consider the reversed difference.

5. KL divergence, $D_{K L}\left(f_{A B} \| f_{C D}\right)$ : Each term within the summation in the definition for KL divergence given by Eq. 3 can be considered as the contribution of a pattern $v$ towards the total divergence between the two distributions. This is an asymmetric measure. We use it to compute an asymmetric feature function. Here, we have used the notation $D_{K L}(\cdot \| \cdot)$ to denote the contribution of a pattern $v$ towards the total divergence. Note that $f_{A B}$ and $f_{C D}$ are not distributions.

6. Reverse KL divergence, $D_{K L}\left(f_{C D} \| f_{A B}\right)$ : Same as the above, except we consider the reverse of the two pattern frequencies.

There are $5+6=11$ different feature functions. We use those feature functions to construct feature vectors for given two pairs of words. Next, we train a binary support vector machine as described in Sect. 3.3. We use five popular kernels in our experiments: linear, quadratic (degree = 2 ), cubic (degree $=3$ ), Radial Basis Functions (RBF), and the Sigmoid kernel. To evaluate the effect of different kernels with different feature functions, we use each feature function separately with each kernel function to train and test on SAT word analogy questions. We use svmlight ${ }^{\dagger \dagger}$ as the SVM implementation in our experiments. We do not tune any of the parameters in SVM including the kernel parameters. All parameters are set to their default values in svmlight. To avoid any bias towards the difference in the range of absolute values of features, we normalize each feature to range $[0,1]$ by dividing from the maximum value of that feature. There are 374 word analogy questions in the SAT dataset. Each question typically has 5 candidate answer pairs, whereas for a small number of questions there are only 4 candidate answer pairs. There is only one correct answer word pair for each SAT word analogy question. We randomly select 50 questions for testing and the remaining $374-50=324$ questions are used as training data. Experimental results for are presented in Table 2.

From Table 2, we see that the combination of multi- plicative feature function $\left(f_{A B} \times f_{C D}\right)$ with RBF kernel produces the maximum SAT score of $58 \%$ on the test dataset. Among all asymmetric feature functions, the KL divergence $\left(D_{K L}\left(f_{A B} \| f_{C D}\right)\right)$ reports the maximum SAT score when used with the cubic kernel. The performance of $f_{A B} \times f_{C D}$ and $D_{K L}\left(f_{A B} \| f_{C D}\right)$ are comparable across different kernel functions, except in Sigmoid kernel where KL divergence perform poorly. Considering the fact that SVMs are sensitive to the kernel parameters, we believe that the poor performance observed with the Sigmoid kernel is a result of the suboptimal kernel parameter values. All kernels perform consistently well when used with multiplicative feature function even without any parameter tuning. Therefore, we can conclude that multiplicative feature function is robust against different kernel choices when used in SVMs.

It is noteworthy that both subtraction and division of feature values perform poorly with all kernel functions. Considering the fact that random guessing on SAT dataset yields an SAT score of $20 \%$ the performance of those asymmetric feature functions is not significantly different from the random baseline. KL divergence-based feature function clearly outperforms all other asymmetric feature functions when used with any of the kernels considered in this experiment. This shows that although asymmetric feature functions are useful to detect relational similarity, naively combining features to produce asymmetric feature functions is not desirable. It is particularly interesting to note that the KL divergence $\left(D_{K L}\left(f_{A B} \| f_{C D}\right)\right)$ outperforms its counterpart, the reverse KL divergence $\left(D_{K L}\left(f_{C D} \| f_{A B}\right)\right)$. This result shows that in SAT word analogy questions we must take into consideration the fact that we are comparing a single question word pair with multiple candidate answer pairs. Lexical patterns that occur in a question word pair (i.e. $(A, B)$ ) define the relation that is considered by that question. In $D_{K L}\left(f_{A B} \| f_{C D}\right)$, the log ratios are weighted by the probabilities of patterns that occur in the question word pair. Therefore, divergences from the patterns that occur frequently in the question word pair are weighted higher than those that occur less frequently in question word pair.

From Table 2, we can see that quadratic and cubic kernels outperform the linear kernel in the best symmetric (multiplicative) and asymmetric (KL divergence) feature functions. Unlike the linear kernel, which considers each feature independently, polynomial kernels consider combinations of features. Therefore, the superior performance reported by polynomial kernels against the linear kernel suggests that the lexical patterns that we use as features are not independent. This can be explained considering the fact that there exist multiple lexical patterns that represent the same semantic relation.

\subsection{Comparison against Previous Work}

Table 3 summarizes various relational similarity measures proposed in previous work. All algorithms in Table 3 are

\footnotetext{
${ }^{\dagger \dagger} \mathrm{http}: / /$ www.cs.cornell.edu/People/tj/svm_light/
} 
Table 3 Comparison against previous work.

\begin{tabular}{|c|c|c|c|c|c|}
\hline & Algorithm & score & & Algorithm & score \\
\hline 1. & Phrase Vectors & 38.2 & 2. & Thesaurus Paths & 25 \\
\hline 3. & Synonym & 20.7 & 4. & Antonym & 24 \\
\hline 5. & Hypernym & 22.7 & 6. & Hyponym & 24.9 \\
\hline 7. & Meronym:substance & 20 & 8. & Meronym:part & 20.8 \\
\hline 9. & Meronym:member & 20 & 10. & Holonym:substance & 20 \\
\hline 11. & Holonym:member & 20 & 12. & Similarity:dict & 18 \\
\hline 13. & Similarity:wordsmyth & 29.4 & 14. & Combined [6] & 45 \\
\hline 15. & Symmetric Features [5] & 40.1 & 16. & Proposed (RankSVM) & 46.9 \\
\hline 17. & WordNet [8] & 42.8 & 18. & VSM [7] & 47.1 \\
\hline 19. & Pertinence [14] & 53.5 & 20. & LRA [2] & 56.1 \\
\hline
\end{tabular}

evaluated on the same SAT analogy questions. Score (given by Formula 1) is the percentage of correctly answered questions to the total number of questions (374) in the dataset. An SAT question typically contain 5 choices. Therefore, a random guessing algorithm would obtain a score of 20 . The score reported by average senior high-school student is about 57 [7]. We performed 5-fold cross validation on SAT questions to evaluate the performance of the proposed method using the multiplicative feature function and the RBF kernel, which we found to be the best combination in Table 2. We do not tune any parameters in the RBF kernel nor the SVM. The results reported in Table 2 must not be directly compared against the SAT scores in Table 3 because in Table 2 we do not perform a cross-validation over the entire 374 questions instead use a randomly selected test set of 50 questions for efficiency reasons when training with a wide range of kernel functions and feature functions.

The first 13 algorithms were proposed by Turney et al. [6], in which they combined these modules using a weight optimization method. For given two word pairs, the phrase vector (row 1) algorithm creates a vector of manually created pattern-frequencies for each word-pair and compute the cosine of the angle between the vectors. Algorithms in rows 2-11 use WordNet to compute various relational similarity measures based on different semantic relations defined in WordNet. Similarity:dict (row 12) and Similarity:wordsmith (row 13) respectively use Dictionary. com and Wordsmyth . net to find the definition of words in wordpairs and compute the relational similarity as the overlap of words in the definitions. The proposed method outperforms all those 13 individual modules reporting a score of 46.9 , which is comparable to the combined approach which has an SAT score of 45.

The Symmetric Features (row 15) [5] only uses symmetric feature functions when generating a feature vector to represent two pairs of words. However, as we already discussed in Table 2, asymmetric feature functions such as the KL divergence measure are more adapted for answering SAT word analogy questions where there exist a distinction between the question word pair and the candidate answer word pairs. Moreover, the number of lexical patterns used in our proposed method is much larger $(48,253)$ than the Symmetric Features method which uses a relatively small set of 9,980. Therefore, we can represent a semantic relation that exists between two words using a richer feature representation.

The proposed method outperforms the WordNet-based relational similarity measure [8]. One limitation of the WordNet-based relational similarity measure is that it cannot compute the relational similarity between word pairs where at least one of the four words is not in the WordNet. Because named entities are not well-covered by WordNet, we believe that the proposed relational similarity measure can be useful when computing relational similarity between pairs that involve named entities. In future work, we intend to evaluate the proposed method using named entities.

The SAT score reported by the proposed method is comparable to the VSM method. However, the proposed method is outperformed by the LRA method. One reason for this might be that we only consider co-occurrences of patterns in snippets, whereas VSM, pertinence and LRA use co-occurrences of patterns in a large corpus. Therefore, it is interesting to explore the possibility of supervised learning approaches to measuring relational similarity using cooccurrences in a larger corpus. Although there are multiple incorrect answers in an SAT question, they are not ranked by their degree of relational similarity in the SAT benchmark dataset. If we can obtain some ranking information for the incorrect answers, then we can use that to further guide the learning process. One possibility is to rank each incorrect answer according to the number of times it was selected by examinees. If many examinees select an incorrect answer, then it is likely that it is relationally similar to the question word pair although it is not the correct answer to the question.

\section{Conclusion}

We proposed a supervised binary classification approach to measure the relational similarity between two given word pairs. First, we represented a word pair using a feature vector where we select lexical patterns that co-occur with that word pair in text snippets retrieved from a Web search engine. Second, we use the SAT word analogy dataset to generative positive (relationally similar) and negative (relationally dissimilar) training instances to train a binary support vector classifier. The distance from the decision hyperplane is transformed into a posterior probability to measure the degree of relational similarity between two word pairs. Our proposed method achieved an SAT score of 46.9 on a bench- 
mark dataset of 374 SAT word analogy questions. In our future work, we intend to study the effectiveness of the proposed method to measure the relational similarity between named entities.

\section{References}

[1] V. Natase and S. Szpakowicz, "Exploring noun-modifier semantic relations," Proc. fifth int'l workshop on computational semantics (IWCS-5), pp.285-301, 2003.

[2] P. Turney, "Similarity of semantic relations," Computational Linguistics, vol.32, no.3, pp.379-416, 2006.

[3] S. Banerjee and T. Pedersen, "Extended gloss overlaps as a measure of semantic reladeness," Proc. IJCAI'03, pp.805-810, 2003.

[4] V. Vapnik, Statistical Learning Theory, Wiley, Chichester, GB, 1998.

[5] D. Bollegala, Y. Matsuo, and M. Ishizuka, "Www sits the sat: Measuring relational similarity on the web," ECAI 2008: Proc. 18th European Conference on Artificial Intelligence, July, 2008, Patras, Greece: Including Prestigious Applications of Intelligent, pp.333337, 2008.

[6] P. Turney, M. Littman, J. Bigham, and V. Shnayder, "Combining independent modules to solve multiple-choice synonym and analogy problems," Proc. RANLP'03, pp.482-486, 2003.

[7] P. Turney and M. Littman, "Corpus-based learning of analogies and semantic relations," Mach. Learn., vol.60, pp.251-278, 2005.

[8] T. Veale, "Wordnet sits the sat: A knowledge-based approach to lexical analogy," Proc. 16th European Conference on Artificial Intelligence (ECAI'04), pp.606-612, 2004.

[9] D. Bollegala, Y. Matsuo, and M. Ishizuka, "Measuring the similarity between implicit semantic relations from the web," WWW 2009, pp.651-660, 2009.

[10] J. Pei, J. Han, B. Mortazavi-Asi, J. Wang, H. Pinto, Q. Chen, U. Dayal, and M. Hsu, "Mining sequential patterns by pattern-growth: the prefixspan approach,” IEEE Trans. Knowl. Data Eng., vol.16, no.11, pp.1424-1440, 2004.

[11] G. Salton and C. Buckley, Introduction to Modern Information Retreival, McGraw-Hill Book Company, 1983.

[12] J. Platt, "Probabilistic outputs for support vector machines and comparison to regularized likelihood methods," Advances in Large Margin Classifiers, pp.61-74, 2000.

[13] C.D. Manning and H. Schütze, Foundations of Statistical Natural Language Processing, The MIT Press, Cambridge, Massachusetts, 2002.

[14] P. Turney, "Expressing implicit semantic relations without supervision,” Proc. Coling/ACL'06, pp.313-320, 2006.

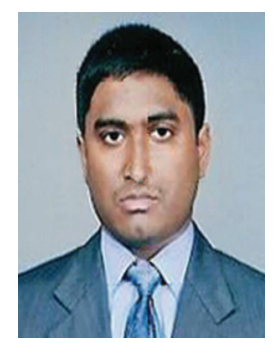

Danushka Bollegala received his BS, MS and $\mathrm{Ph} . \mathrm{D}$. degrees from the University of Tokyo, Japan in 2005, 2007, and 2009. He is currently an assistant professor at the Graduate School of Information Science and Technology, the University of Tokyo. His research interests are natural language processing, Web mining and artificial intelligence.

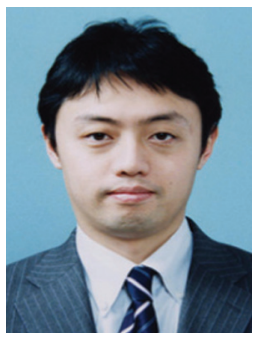

Yutaka Matsuo is an associate professor at Graduate School of Engineering, the University of Tokyo, Japan. He received his BS, MS, and $\mathrm{Ph} . \mathrm{D}$. degrees from the University of Tokyo in 1997, 1999, and 2002. He joined National Institute of Advanced Industrial Science and Technology (AIST) from 2002 to 2007 . He is interested in social network mining, text processing, and semantic web in the context of artificial intelligence research.

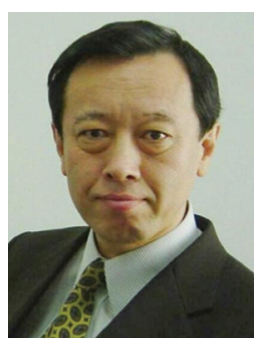

Mitsuru Ishizuka is a professor at Graduate School of Information Science and Technology, the University of Tokyo, Japan. Previously, he worked at NTT Yokosuka Laboratory and Institute of Industrial Science, the University of Tokyo. He was also a visiting associate professor at Purdue University during 1980-1981. He received his BS and Ph.D. degrees in electric engineering from the University of Tokyo in 1971 and 1976, respectively. His research interests include artificial intelligence, Web intelligence, semantic computing, and multimodal lifelike agents. He is the past president of JSAI (Japanese Society for Artificial Intelligence). 